testing and treatment for FSW at different annual coverage levels for 3 years, in comparison to using a high sensitivity lab-based test. Because of evidence for $\mathrm{Tp}$ rebounding following treatment, the impact of the intervention after it has ended was evaluated.

Results The model projected the 3-year impact of annually screening $40 \%$ or $80 \%$ of FSWs with a rapid Tp test (sensitivity $87 \%)$ would result in a $46 \%(43-54 \%)$ or $69 \%(66-77 \%)$ relative reduction in $\mathrm{Tp}$ prevalence in this setting, respectively. The corresponding decrease in Tp prevalence among clients was estimated to be 2 to 3 -fold less but more infections were averted among them. The Asbtract P1-S2.17 figure 1 shows that for a lab-based testing strategy to achieve the same impact as using a rapid test with $87 \%$ sensitivity would require a return rate of $92 \%$ or $98 \%$ if the delay in treatment was 1 or 2 weeks, respectively. After screening ceases, the model projects the percentage of infections averted would continue increasing for a number of years in this setting.

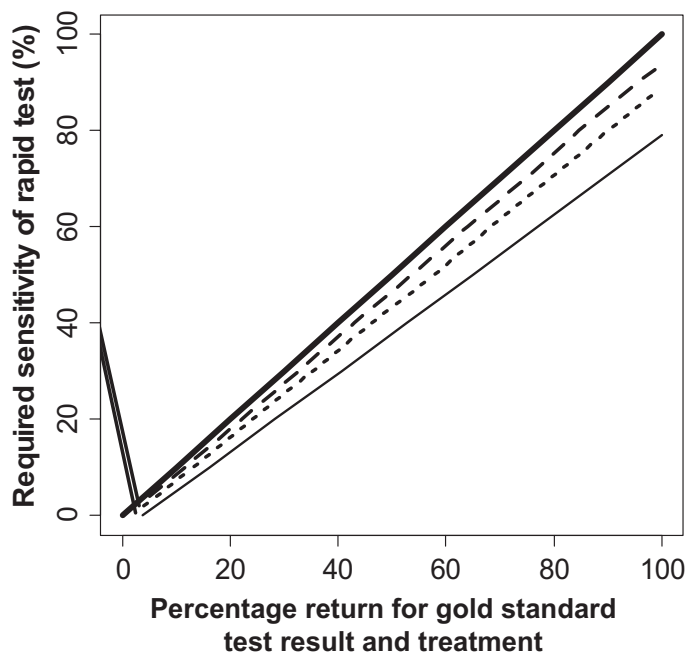

Asbtract P1-S2.17 Figure 1 Required return rate for lab-based gold standard test to have same impact as a rapid test for different delays in treatment. Different lines are for different delay periods between testing and treatment for the lab test - thick line, 0 weeks; dashed line, 1 week; dotted line, 2 weeks; narrow line, 4 weeks.

Conclusions In a moderate Tp prevalence setting, a rapid test point of care intervention could have considerable impact on Tp transmission. Unless there is very low sensitivity for the rapid test and a very high return rate for the lab-based method of screening, the rapid test intervention will provide a greater relative reduction in $\mathrm{Tp}$ prevalence.

\section{P1-S2.18 SYPHILIS PREVALENCE AND RELATED RISK FACTORS AMONG FEMALE WORKERS AT SOCIAL VENUES IN LIUZHOU, CHINA}

doi:10.1136/sextrans-2011-050108.75

${ }^{1} \mathrm{~J} \mathrm{Li},{ }^{1} \mathrm{X}$ S Chen, ${ }^{2,3} \mathrm{~A}$ Gandhi, ${ }^{2,3} \mathrm{C}$ Suchindran, ${ }^{2,3} \mathrm{~S}$ Weir. ${ }^{1}$ The National Center for STD Control and the Chinese Academy of Medical Sciences Institute of Dermatology, Nanjing, China; ${ }^{2}$ The Gillings School of Global Public Health, University of North Carolina, Chapel Hill, USA; ${ }^{3}$ The Carolina Population Center, University of North Carolina, , Chapel Hill, USA

Background In the context of rapid urbanisation and economic growth in China, places where social mixing intersects with commercial activities could play an important role in the formation of new partnerships and spread of sexually transmitted infections (STIs) including HIV. People who work at such venues may be at higher risk of STIs. A study was conducted from October 2009 through February 2010 in Liuzhou, China to estimate the prevalence of syphilis and related risk factors among females working at places where people go to meet new sexual partners.

Methods Using a two-stage venue-based sampling method called PLACE, data were collected through interviews with 656 females nested within 36 venues with a minimum of five females per venue. A venue was classified as a "sex venue" if the manager or at least one of the females reported solicitation of commercial sex at the venue in the past 4 weeks. All respondents were screened for syphilis onsite using a rapid treponemal-specific test which represents lifetime syphilis infection acquired at anytime. Multi-level regression modelling was used to examine individual and venue correlates of syphilis infection and to account for clustering.

Results 23 of the 36 venues were classified as sex venues. Among all females, those working at sex venues and at venues with no report of sex work, the weighted lifetime syphilis prevalence was $5.8 \%$, $16.3 \%, 0.5 \%$ respectively and the reported average number of new sexual partners in the past 4 weeks was $1.5,4.4,0.1$ respectively. After adjusting for other individual characteristics and clustering, two venue-level factors, that is working at sex venues (AOR 24.0, $95 \%$ CI 2.5 to 227.6 ), working in venues located in rural counties (AOR 3.6, 95\% CI 1.2 to 10.9), and two individual correlates, ie, selfreport of sex work (AOR 2.3, 95\% CI 1.0 to 5.3) and being in elder age groups (AOR 7.0, 95\% CI 1.5 to 32.2 ) were significantly associated with risk of lifetime syphilis infection.

Conclusions Females working at social venues had a high rate of new sexual partner acquisition and a high prevalence of lifetime syphilis infection. Females working at sex venues or venues located in rural counties were at relatively high risk of syphilis infection, despite whether they reported engaging in sex work. An intervention strategy focused on the places where social mixing occurs rather than risk group status may contribute to the prevention and control of HIV/STIs in China.

\section{P1-S2.19 UNPROTECTED FELLATIO AND PHARYNGEAL GONORRHOEA IN SYDNEY SEX WORKERS}

doi:10.1136/sextrans-2011-050108.76

${ }^{1} \mathrm{P} J$ Read, ${ }^{2} \mathrm{H}$ Wand, ${ }^{2} \mathrm{R}$ Guy, ${ }^{1} \mathrm{~A}$ McNulty, ${ }^{2} \mathrm{~B}$ Donovan. ${ }^{1}$ Sydney Sexual Health Centre, Sydney, Australia; ${ }^{2}$ National Centre in HIV Epidemiology and Clinical Research, Sydney, Australia

Background Sex workers are a priority population in the WHO sexually transmitted infection (STI) global strategy. In Australia, condom use for vaginal sex has remained high over the past decade rates in sex workers with corresponding low rates of STIs. In recent years, there have been anecdotal reports of Sydney sex workers increasingly reporting unprotected fellatio with clients which has coincided with an increase in cases of pharyngeal gonorrhoea in these women. The aim of this study was to investigate the extent and predictors of inconsistent condom use for fellatio at work, and rates of pharyngeal gonorrhoea among sex workers attending a large sexual health clinic.

Methods All female sex workers reporting fellatio at work seen at the Sydney Sexual Health Centre from May 2009 to January 2011 were included. Demographic data, risk behaviours and STI diagnoses were extracted from the clinic database. Pharyngeal gonorrhoea cultures were collected routinely. Multivariate logistic regression analysis was used to determine predictors of inconsistent condom use for fellatio at work.

Results There were 1539 sex workers seen during the study period-most $(n=1142,74 \%)$ worked in brothels and $24 \%$ reported inconsistent condom use for fellatio at work. Significant independent predictors of reporting inconsistent condom use for fellatio at work were being a new client at our clinic (compared to an 